Article

\title{
Thermochemical Wear of Single Crystal Diamond Catalyzed by Ferrous Materials at Elevated Temperature
}

\author{
Lai Zou ${ }^{1, *}$, Yun Huang ${ }^{1}$, Ming Zhou ${ }^{2}$ and Guijian Xiao ${ }^{1}$ \\ 1 State Key Laboratory of Mechanical Transmission, Chongqing University, Chongqing 400044, China; \\ yunhuang@samhida.com (Y.H.); xiaoguijian@cqu.edu.cn (G.X.) \\ 2 School of Mechanical and Electrical Engineering, Harbin Institute of Technology, Harbin 150001, China; \\ zhouming@hit.edu.cn \\ * Correspondence: zoulai@cqu.edu.cn; Tel.: +86-23-6766-9883; Fax: +86-23-6766-9663
}

Academic Editor: Yuri N. Palyanov

Received: 25 March 2017; Accepted: 17 April 2017; Published: 19 April 2017

\begin{abstract}
Single crystal diamond has been recognized as the optimal tool material in ultra-precision machining. However, the excessive tool wear prevents it from cutting ferrous materials. This paper conducts a series of thermal analysis tests under the conditions of different gas atmospheres, heating temperatures, crystallographic planes and workpiece materials, in order to clarify the details of thermochemical wear of diamond catalyzed by iron at elevated temperature. Raman scattering analysis was performed to identify the transformation of diamond crystal structure. Energy dispersive X-ray analysis was used to detect the change in chemical composition of the work material. X-ray photoelectron spectroscopy was adopted to confirm the resultants of interfacial thermochemical reactions. The experimental results revealed that the diamond wear included the graphitization, diffusion and oxidation. Temperature was considered as the key factor affecting these wear mechanisms. The initial graphitization temperatures of diamond catalyzed by iron under different conditions were obtained, and the graphitized degree relied heavily on the crystallographic plane while being insensitive to the workpiece material. The diffusion wear rule was preliminarily achieved by the established prediction model of the carbon atoms diffusing into the iron lattice, and the types and resultants of interfacial chemical reactions were deduced.
\end{abstract}

Keywords: diamond; thermochemical wear; graphitization; diffusion; oxidation; ferrous materials; chemical reaction; thermal analysis

\section{Introduction}

Natural single crystal diamond has been widely used in ultra-precision machining of nonferrous metals owing to its outstanding mechanical and physicochemical properties [1,2]. However, cutting of ferrous metals with diamond tools has not been successful in application to date as the tool wear rate is severe [3,4]. Meanwhile, with the requirement for high precision and complex moulds to be made of ferrous materials increasing greatly in recent years, it is difficult to guarantee the demands for machining efficiency and machining accuracy using the conventional processes [5]. Therefore, it is necessary to investigate the tool wear of single crystal diamond in the cutting operation of ferrous metals.

Several technological methods have been proposed for overcoming this serious tool wear issue: turning of medium carbon steels in different carbon-saturated atmospheres [6,7]; turning of stainless steels in a low temperature environment [8]; adopting the ultrasonic vibration assisted turning technique to decrease the cutting force and temperature for controlling tool wear and obtaining optical-quality surface $[9,10]$; restraining the chemical reactions between carbon atoms and iron 
atoms at high temperature by surface modification of the diamond tool and workpiece materials [11]; and proposing an ion-shot coolant method for micro-cutting of ferrous materials [12].

Although these auxiliary processing methods mentioned above could decrease the diamond tool wear to various degrees, they have not met the requirements of industrial application, as rather less attention has been paid to the wear mechanisms of a single crystal diamond tool. Thornton et al. [13] performed experimental investigations on turning medium carbon steels under the conditions of different gas atmospheres and cutting speeds. The results revealed that diamond graphitization was the main cause for tool wear, which was attributed to the local high temperature, the continuous clean surface with high chemical activity produced by cutting and the oxygen molecules in the air. In addition, it was pointed out that there was no diamond diffusion wear in the cutting process. Paul et al. [14] found that ferrous metals were classified as non-diamond turnable materials ascribed to the unpaired numbers of $d$-shell electrons of the iron atoms, and the wear rate was proportional to the number of unpaired $d$ electrons. Shimada et al. $[15,16]$ suggested that the tool wear had a strong correlation with thermochemical reactions between diamond and ferrous metals based on the results of static erosion tests. However, there was not much detailed information about the functional mechanism of each wear form. Narulkar et al. [17] utilized molecular dynamics and realistic interaction potentials as the first direct evidence that the structure of diamond at the cutting edge initially transformed from diamond cubic into hexagonal graphite in the presence of iron. Subsequently, the graphitic carbon diffused into the iron surface. Zou et al. [18] chiefly investigated the graphitization wear of single crystal diamond against ferrous metals by frictional wear experiments. The results showed that the effect of mechanical force on tool wear should not be ignored and the diamond graphitized degree was proportional to mechanical force and sliding velocity. There was, as yet, no consistent explanation of the diamond tool wear mechanism, which was a limitation on the development of the machining process.

In view of this, a series of thermal analysis experiments simulating the wear process of single crystal diamond were proposed in this work, in order to investigate the thermochemical wear of diamond surface catalyzed by iron at elevated temperature under different gas atmospheres, for thoroughly understanding the individual and intensive diamond tool wear mechanism in machining of ferrous materials.

\section{Materials and Methods}

\subsection{Sample Preparation}

Similar sized natural single crystal diamonds were used in these tests, which were polished with crystallographic plane (110) and (100), respectively. Four kinds of stainless steels with different carbon content were selected as the workpiece materials, which were widely used in mechanical and electronic fields. Table 1 presents the chemical compositions of each work material. These ferrous materials were cut by wire electrical discharge machining to the diameter and thickness of 4 , and $0.2 \mathrm{~mm}$, respectively. It is worth mentioning that the diamonds and steels were cleaned carefully in an ultrasonic vessel with acetone before each thermal analysis experiment.

Table 1. Chemical compositions of the work samples used in tests.

\begin{tabular}{cccccccccc}
\hline \multirow{2}{*}{ Workpiece } & \multicolumn{8}{c}{ Chemical Compositions $(\omega \%$, Balance Fe $)$} \\
\cline { 2 - 10 } & $\mathbf{C}$ & Mn & Si & S & P & Ni & Cr & Ti & Mo \\
\hline 0Cr18Ni9 & 0.045 & 1.13 & 0.35 & 0.003 & 0.033 & 8.04 & 17.10 & - & 0.03 \\
1Cr18Ni9Ti & 0.07 & 1.21 & 0.37 & 0.01 & 0.03 & 8.07 & 17.99 & 0.40 & - \\
2Cr13 & 0.27 & 0.39 & 0.56 & 0.01 & 0.02 & 0.14 & 12.40 & - & - \\
3Cr13 & 0.29 & 0.21 & 0.89 & 0.004 & 0.021 & 0.21 & 12.47 & - & - \\
\hline
\end{tabular}


Subsequently, the diamond polished with the designated crystallographic plane was set at the bottom surface of an aluminum oxide crucible in contact with the ferrous specimen. Figure 1 shows the schematic model of the thermal corrosion between diamond and steel specimen.

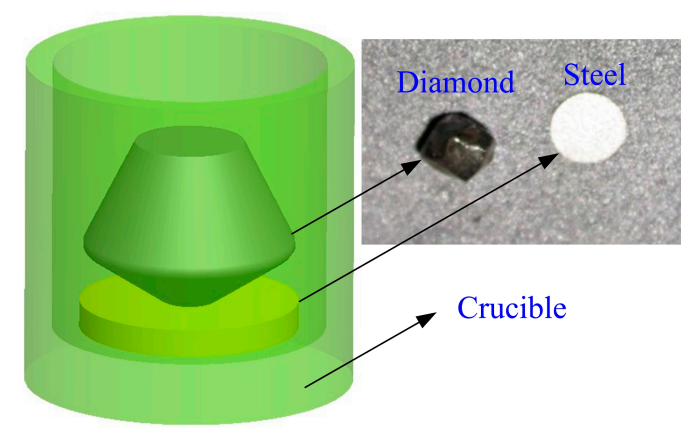

Figure 1. Schematic model of thermal analysis between diamond and steel specimen.

\subsection{Characterization}

\subsubsection{Experimental Procedure}

In order to accurately describe the thermochemical wear process of the single crystal diamond catalyzed by ferrous materials at elevated temperature, thermal analysis experiments were performed using a TGA1600 simultaneous thermal analyzer with two functions, including differential thermal analysis (DTA) and thermal gravimetric (TG). Figure 2 displays the working principle of this thermal analysis technique. In this work, the samples that consisted of diamond and steel were heated in a designated temperature range, and the heating rate was set to $10 \mathrm{~K} / \mathrm{min}$. Moreover, the argon or air continuously penetrated into the crucible with a flow rate of $40 \mathrm{~mL} / \mathrm{min}$ during the heating process. The total mass of the samples was approximately $100 \mathrm{mg}$. Table 2 presents the parameters of the thermal analysis experiments.

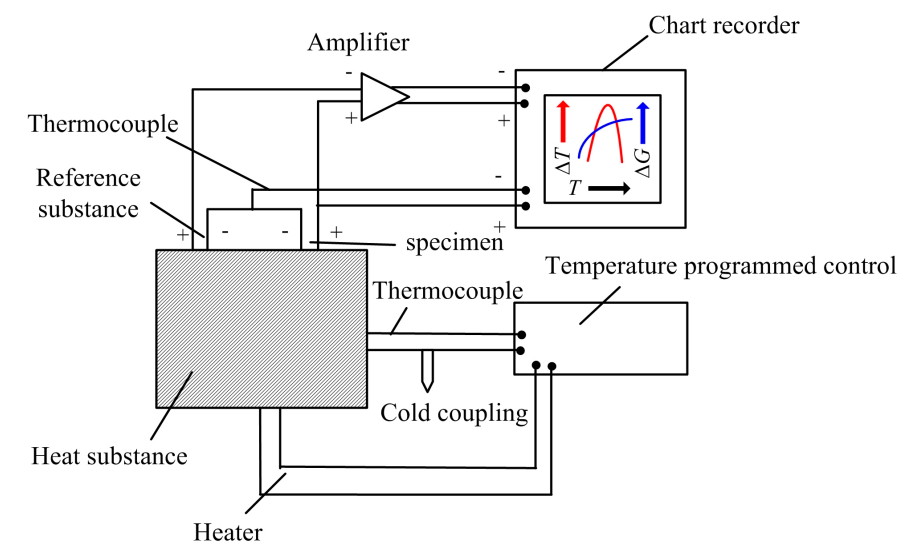

Figure 2. Working principle of the thermal analysis technique.

Table 2. Parameters of the thermal analysis experiments.

\begin{tabular}{cc}
\hline Parameters & Value \\
\hline Heating range $(\mathrm{K})$ & $293 \sim 1273 / 1573$ \\
Heating rate $(\mathrm{K} / \mathrm{min})$ & 10 \\
Gas flow rate $(\mathrm{mL} / \mathrm{min})$ & 40 \\
Gas atmosphere & argon $/$ air \\
Polished plane of diamond & $(100) /(110)$ \\
Workpiece materials & 0Cr18Ni9/1Cr18Ni9Ti/2Cr13/3Cr13 \\
\hline
\end{tabular}




\subsubsection{Analytic Technique}

Raman spectroscopy (RS) was considered as the most effective tool to distinguish the structural changes of the carbon cluster [19]. The crystal structure transforming from tetrahedral diamond to regular hexagonal graphite was identified by the BWS435-532 Raman spectrometer (B\&W Tek, Newark, NJ, USA) in this work. The $532 \mathrm{~nm}$ wavelength of an Ar ion laser can be focused onto the sample surface down to a spot size of $1 \mu \mathrm{m}^{2}$ with the system resolution of $5 \mathrm{~cm}^{-1}$, and these parameters were kept unanimous for detecting the diamond worn surfaces in all the tests.

The thermal corrosion surface morphology of the samples was examined using the FEI Quanta200 scanning electron microscope (SEM) (FEI, Hillsboro, OR, USA). The changing chemical composition of the ferrous specimen before and after the test could be surveyed by the energy dispersive X-ray (EDX) analysis, in order to study the influence of correlative factors on the diffusion wear of the carbon atoms in single crystal diamond continuously diffused onto the corrosion surface of the ferrous materials.

ESCALAB250 X-ray photoelectron spectroscopy (XPS) (Thermo Fisher Scientific, Waltham, MA, USA) was used to detect the chemical compounds generated at the thermal corrosion interface between diamond and steel specimen for the purpose of confirming the type of thermochemical reactions. It was the fundamental research for further studying the diamond oxidation wear. The energy scanning range of this instrument was $0 \sim 5000 \mathrm{eV}$, continuously adjustable in the range of $1 \sim 400 \mathrm{eV}$ with the step size of $1 \mathrm{eV}$, and the beam spot area was in the range of $900 \sim 20 \mu \mathrm{m}$ with the step size of $50 \mu \mathrm{m}$.

\section{Results and Discussion}

\subsection{Thermal Analysis Results}

Initially, the thermochemical changes of the experimental samples during the heating process were qualitatively analyzed in accordance with the characteristics of the DTA curves, and then the existence and corresponding temperature range of these changes were further concluded in combination with the features shown in the TG curves. Ultimately, the thermal corrosion process of the single crystal diamond catalyzed by ferrous materials at high temperature could be precisely described using the related theory and analytic technique.

Figure 3 shows the DTA and TG curves of the specimens heated in different gas environments from room temperature to $1573 \mathrm{~K}$ and $1373 \mathrm{~K}$. It can be found that an obvious exothermic peak emerged corresponding to the temperature in the vicinity of $1383 \mathrm{~K}$ from the DTA curve in Figure 3a. As the transformation of diamond to graphite was an exothermic process in view of the thermodynamics theory, it can be excluded that this phenomenon was caused by oxidation of the experimental specimens in argon atmosphere. Therefore, it could be initially regarded that the emergence of this exothermic peak was due to the phase transition from the diamond structure to the graphite structure. Besides, it was clear that the overall quality of the samples basically did not change, as shown from the TG curve in Figure 3a because no gas escape would be produced by the chemical reaction in this atmosphere.

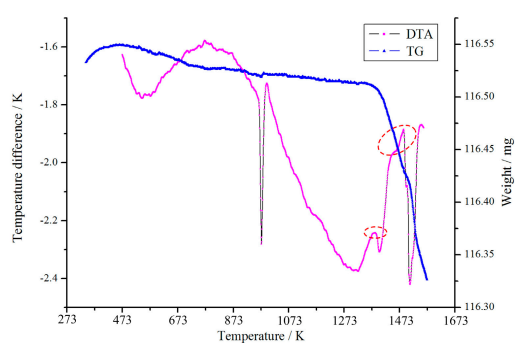

(a)

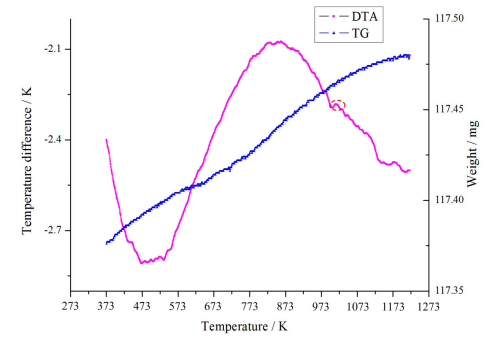

(b)

Figure 3. Differential thermal analysis (DTA) and thermal gravimetric (TG) curves of the specimens heated in different gas environments; the workpiece material was 2Cr13; the diamond crystallographic plane was (100); the temperature range was from $293 \mathrm{~K}$ to $1573 \mathrm{~K}$ and $1273 \mathrm{~K}$. (a) DTA-TG curves in argon; (b) DTA-TG curves in air. 
Moreover, the thermal analysis curves shown in Figure $3 \mathrm{~b}$ were analyzed in accordance with the same method. The emergence of the tiny exothermic peak could be primarily attributed to the diamond graphitization while the temperature was approximately $1013 \mathrm{~K}$. As a series of complex oxidation-reduction reactions might occur at the corrosion interface between diamond and steel in air condition, it would result in an obvious exothermic process and the total weight of the specimens increased.

Figure 4 presents the worn morphology of the single crystal diamonds under the same experimental conditions as shown in Figure 3. It can be clearly seen that the corrosion degree of diamond in argon was far lower than that in air, and part of the steel specimen was attached on the diamond surface under the effect of high temperature. The detailed wear process will be discussed in the following part about the thermochemical wear mechanisms of diamond catalyzed by the ferrous materials.

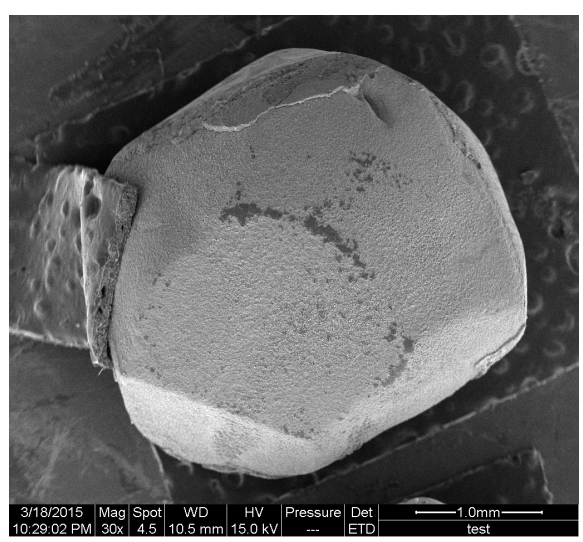

(a)

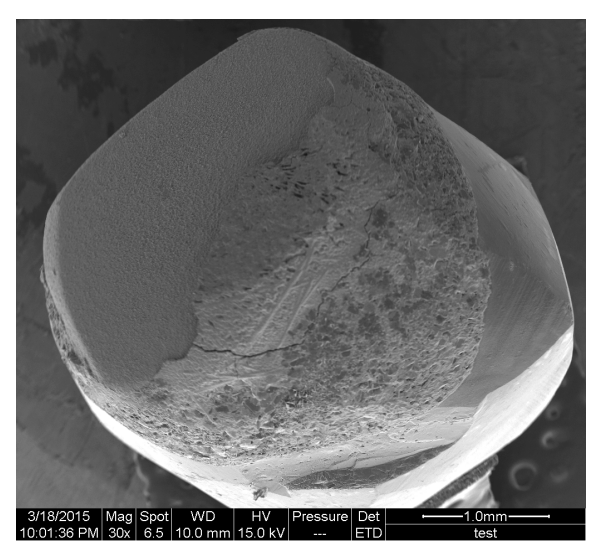

(b)

Figure 4. Worn morphology of the single crystal diamonds corroded by the ferrous materials; the workpiece material was $2 \mathrm{Cr} 13$; the diamond crystallographic plane was (100); the temperature range was from $293 \mathrm{~K}$ to $1573 \mathrm{~K}$ and $1273 \mathrm{~K}$. (a) Worn morphology in argon; (b) Worn morphology in air.

\subsection{Thermochemical Wear Mechanisms}

The thermal corrosion interface between diamond and steel specimen was further analyzed using advanced testing methods mentioned above based on the results of the thermal analysis experiments, and the thermochemical wear mechanisms of single crystal diamond will be studied and revealed in depth from the several subsequent aspects.

\subsubsection{Graphitization Wear}

Figure 5 shows the Raman spectra curves of the diamond corrosion surface. The peaks corresponding to the Raman shift at approximately $1582 \mathrm{~cm}^{-1}$ were attributed to the sp $\mathrm{s}^{2}$ hybridized orbit of graphite, while others corresponding to the Raman shift at approximately $1334 \mathrm{~cm}^{-1}$ were attributed to the $\mathrm{sp}^{3}$ hybridized orbit of diamond [20]. In view of the occurrence of the graphite structure, it can be confirmed that the exothermic process arose due to diamond graphitization. Therefore, the initial temperature of diamond transformed into graphite was found to be $1383 \mathrm{~K}$ and $1013 \mathrm{~K}$ in different gas environments based on iron catalysis. The results showed that the initial temperature of graphitization was much lower than that with no catalysis in inert gas. It was deduced that the catalysis of steel specimens could significantly reduce the graphitization conditions and accelerate the graphitized degree of diamond.

Consequently, graphitization should be considered as a major factor for tool wear in diamond cutting of ferrous metals. In addition, this result can also be taken into account for a new research direction in diamond tool ultra-precision machining of steels in inert gas. Meanwhile, the lower onset temperature of graphitization in air condition was attributed to the existence of oxygen which would cause a large number of oxidation-reduction reactions between the diamond and iron. 


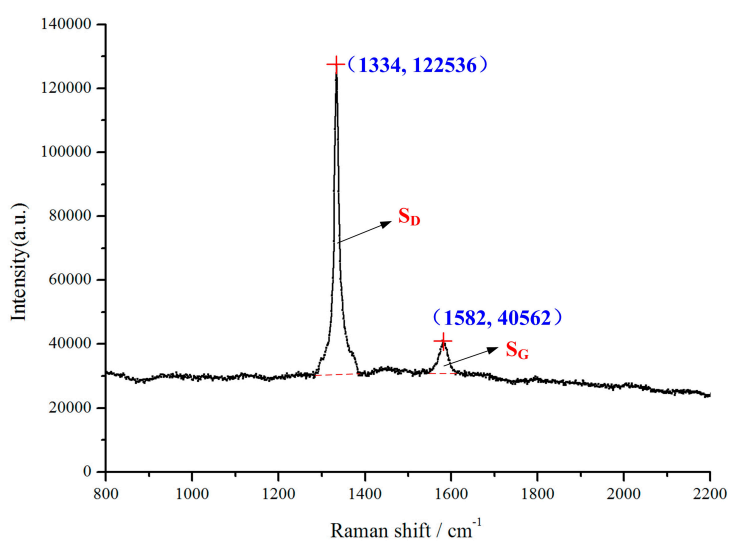

(a)

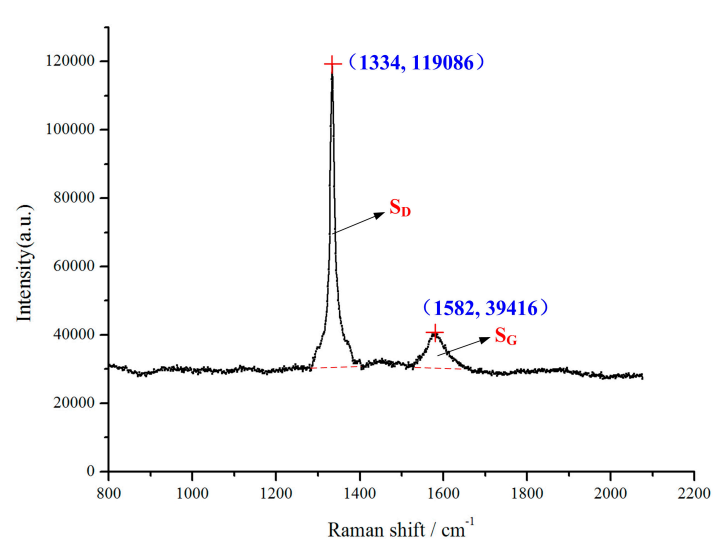

(b)

Figure 5. Raman spectra curves of the diamond surface corroded by the ferrous materials; the workpiece material was $2 \mathrm{Cr} 13$; the diamond crystallographic plane was (100); the temperature range was from 293 K to 1573 K and 1273 K. (a) Raman curves in argon; (b) Raman curves in air.

Equation (1) was proposed based on the principle of Gibbs free energy [21].

$$
\Delta G_{\mathrm{T}}^{\Theta} \leq-1100-4.64 T
$$

where $\Delta G_{\mathrm{T}}^{\Theta}$ and $T$ were Gibbs free energy and heating temperature, respectively. It can obviously be seen that Gibbs free energy would decrease as the temperature was increasing, which results in the diamond graphitized degree being aggravated. This could be used to explicate the appearance of the large exothermic peak when exceeding the onset temperature of diamond graphitization, as shown in Figure 3.

Moreover, Raman scattering intensity, reflected by the area of Raman peak, was proportional to the analyte concentration as indicated in the previous research [19]. Therefore, the graphitized degree of single crystal diamond could be evaluated by the ratio of the graphite peak area $\mathrm{S}_{\mathrm{G}}$ and the diamond peak area $S_{D}$. Table 3 presents the graphitized degree of diamonds under different experimental conditions. The results revealed that the diamond crystallographic plane had an important influence on the graphitization wear, and this can be used as selection criteria of the diamond tool in the actual cutting process. However, the graphitized degree was insensitive to the chemical compositions of the work materials.

Table 3. Graphitized degree of diamonds under different experimental conditions.

\begin{tabular}{cccccc}
\hline \multicolumn{2}{c}{$\mathbf{S}_{\mathrm{G}} / \mathbf{S}_{\mathbf{D}}$ (\%) } & 0Cr18Ni9 & 1Cr18Ni9Ti & 2Cr13 & 3Cr13 \\
\hline \multirow{2}{*}{ Argon } & $(100)$ & 24.6 & 24.3 & 23.8 & 24.1 \\
& $(110)$ & 18.9 & 17.7 & 18.5 & 17.8 \\
\hline \multirow{2}{*}{ Air } & $(100)$ & 45.8 & 45.1 & 44.2 & 44.5 \\
& $(110)$ & 36.5 & 37.4 & 36.8 & 36.2 \\
\hline
\end{tabular}

\subsubsection{Diffusion Wear}

Figure 6 presents the EDX analysis of sample surfaces corroded under the same experimental conditions mentioned above. It can be seen that the carbon content of the steel surface increased obviously after the thermal analysis experiments. It was certain that the diffusion wear occurred at the interface between ferrous material and diamond specimen, especially in argon environment. The minimal diamond diffusion wear that occurred in air condition might be due to the lower maximum heating temperature; the complex chemical reactions also had a certain influence on it. 


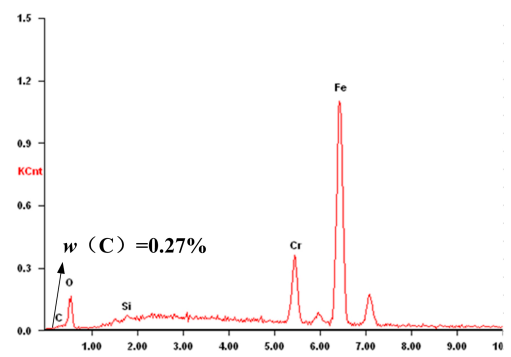

(a)

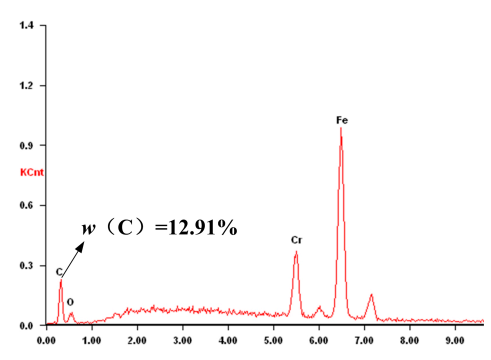

(b)

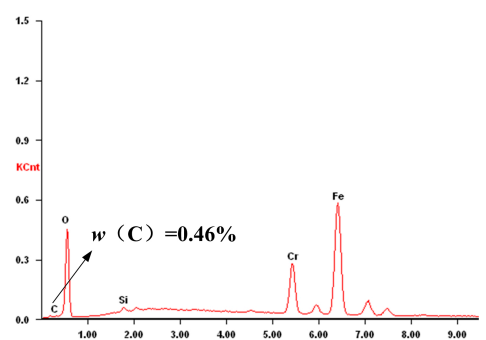

(c)

Figure 6. Energy dispersive X-ray (EDX) analysis curves of the sample surface heated in different gas environments; the workpiece material was 2Cr13; the diamond crystallographic plane was (100); the temperature range was from $293 \mathrm{~K}$ to $1573 \mathrm{~K}$ and $1273 \mathrm{~K}$. (a) Before test; (b) Tested in argon; (c) Tested in air.

Because the iron atoms in ferrous metals had a stronger ability to dissolve carbon than other metal atoms, the carbon atoms in the steel sample could form a certain concentration gradient with diamond. This would cause carbon atoms to become dissociated from the diamond surface and to diffuse into the workpiece material at elevated temperature. However, the experimental results showed that the carbon content of the steel and diamond crystallographic plane had little effect on diffusion wear.

Furthermore, the penetration of carbon atoms into the workpiece surface was primarily considered as a unidimensional diffusion process. In this case, Fick's second law was properly proposed for modeling, as follows, with the hypothesis that the diffusion coefficient was independent of the carbon concentration.

$$
\frac{\partial C(x, \tau)}{\partial \tau}=D \frac{\partial^{2} C(x, \tau)}{\partial x^{2}}
$$

$C(x, \tau)$ was the concentration function of carbon atoms at the diffusion distance $x$ and the diffusion time $\tau$, which needed to be solved to establish the diffusion model. Moreover, the diffusion coefficient $D$ could be decided by the following equation.

$$
D=D_{0} \mathrm{e}^{-Q / R T}
$$

where $D_{0}, Q, R$ and $T$ were the diffusion constant, the diffusion activation energy, the gas constant and the thermodynamic temperature, respectively. It can be seen from this formula that the diffusion coefficient $D$ would generally increase exponentially with the increasing temperature as other parameters remain unchanged. The ultimate diffusion model of carbon atoms that penetrated into the iron crystal lattice was obtained as follows by authors based on relevant assumptions [22].

$$
C(x, \tau)=C_{0}+\left(C_{p}-C_{0}\right) \operatorname{erfc}\left(\frac{x}{2 \sqrt{D \tau}}\right)
$$

where $\operatorname{erfc}(x)$ was the complementary error function used for solving this diffusion model. $C_{0}$ was the solubility of carbon atoms in the ferrous metal at ambient temperature, and $C_{p}$ was the saturated carbon concentration at the specific temperature. It can be seen from this model that the diffusion coefficient and the diffusion time were two crucial factors affecting the distribution laws of carbon concentration, different from the initial carbon concentration of the sample. This tendency was consistent with the results mentioned above. Consequently, the diffusion wear mainly affected by high temperature should be considered as an important wear mechanism of single crystal diamond in machining of ferrous materials, and more attention should be paid to reducing temperature and designing a diffusion barrier at the tool-chip interface to suppress it. The current analytic results could be regarded as the basis for further investigation of diamond diffusion wear in combination with the reasonable experimental studies. 


\subsubsection{Oxidation Wear}

Figure 7 shows the micro-morphology and EDX analysis of the sample surfaces produced by thermal corrosion under the same experimental conditions. As seen in Figure $7 \mathrm{a}, \mathrm{b}$, the result of energy spectrum analysis corresponding to the white block material in micro topography indicated that the carbon atoms diffused from diamond would form a certain carbide with iron atoms in the workpiece material, as a result of no other chemical reactions existing in argon atmosphere. However, the iron-carbon compound was easily decomposed by the effect of elevated temperature. It could be deduced that the possibility of diamond reacting directly with iron to cause tool wear in the cutting operation of ferrous materials was relatively small by combining with the results of thermal analysis experiments.

Unlike the above studies, it can be inferred from the other detection result in Figure 7 that the oxides appeared on the steel surface although at lower temperature in air condition. In order to further verify the existence and types of the iron oxides, the corrosion surface was analyzed by the XPS technique and the results are shown in Figure 8. It can be clearly seen from Figure 8a that the original spectrum curve of O1s coincided well with the fitting line, which could be proof that the other two spectrum curves were reliable. The electron binding energies of the two peaks were $530.14 \mathrm{eV}$ and $531.42 \mathrm{eV}$, respectively. It can be determined that these two curves corresponded to the spectral lines of $\mathrm{Fe}_{x} \mathrm{O}_{y}$ and the $\mathrm{C}-\mathrm{O}$ bond on the basis of relevant analysis data [23], and this result definitely indicated the existence of iron oxides. It was thus that the presence of oxygen would lead to the occurrence of a large number of redox reactions at the interface between experimental samples, resulting in the diamond oxidation wear.

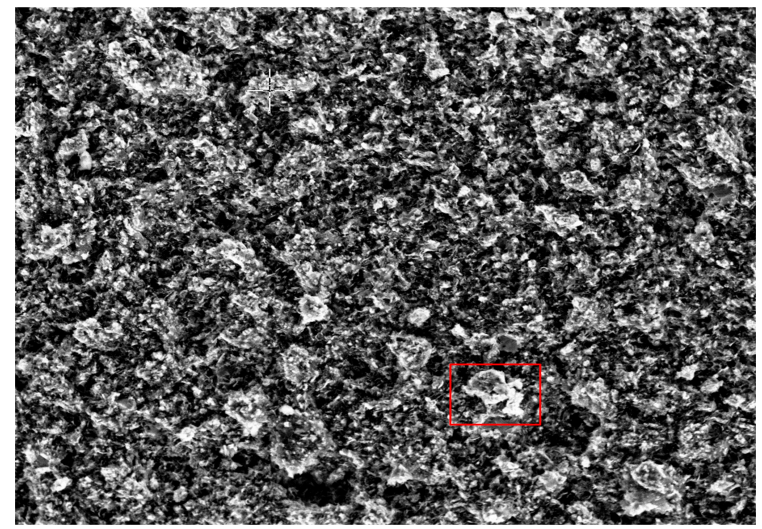

(a)

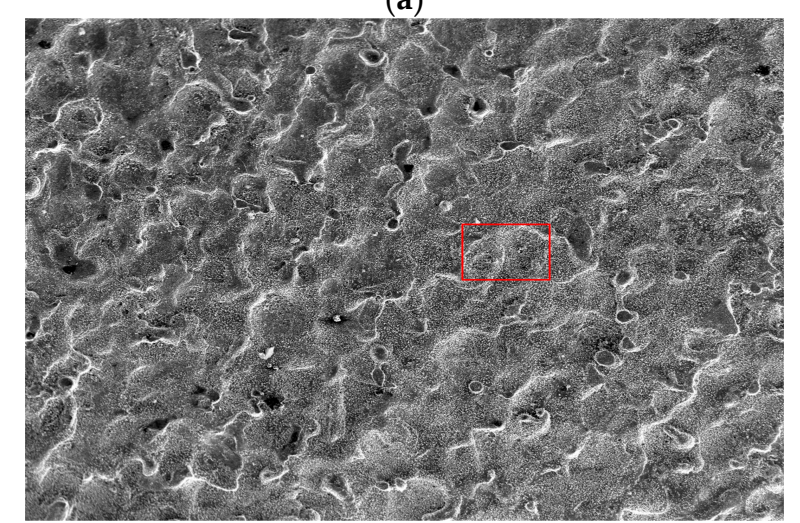

(c)

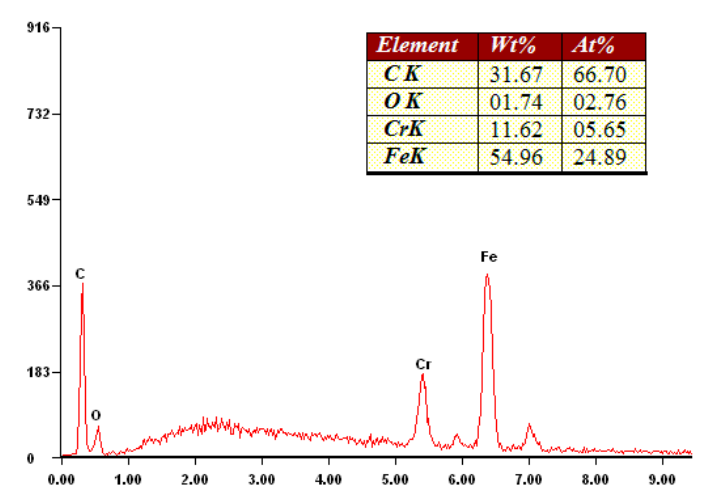

(b)

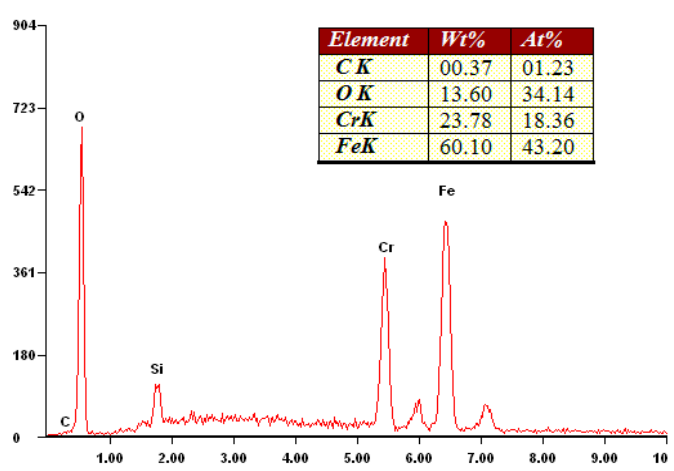

(d)

Figure 7. Micro-morphology and EDX analysis of the sample surfaces corroded under the same experimental conditions. (a) SEM image in argon; (b) EDX analysis in argon; (c) SEM image in air; (d) EDX analysis in air. 


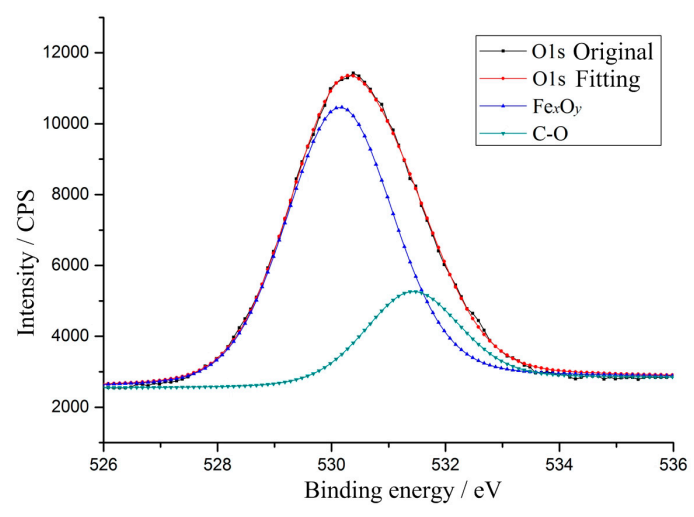

(a)

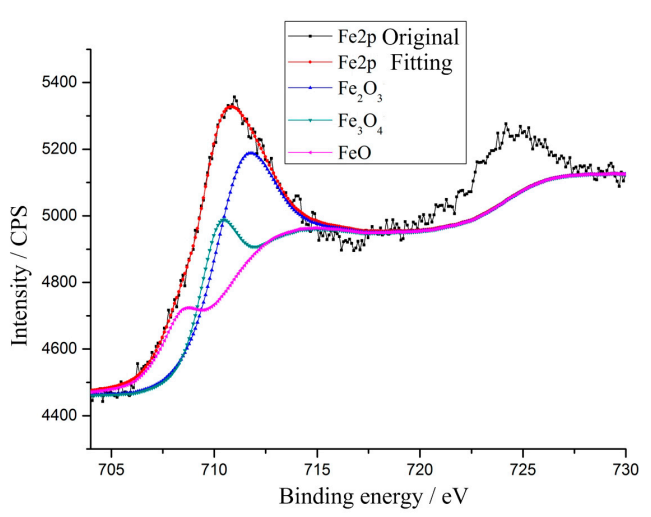

(b)

Figure 8. Electronic spectra curves of $\mathrm{O} 1 \mathrm{~s}$ and $\mathrm{Fe} 2 \mathrm{p}$ characteristic peaks on the sample surface corroded in air condition. (a) Characteristic peaks of O1s; (b) Characteristic peaks of Fe2p.

In addition, the electron binding energies of these three peak curves shown in Figure $8 \mathrm{~b}$ were in the sequence of $708.41 \mathrm{eV}, 710.17 \mathrm{eV}$ and $711.38 \mathrm{eV}$ from small to large, and the corresponding oxides were $\mathrm{FeO}, \mathrm{Fe}_{3} \mathrm{O}_{4}$ and $\mathrm{Fe}_{2} \mathrm{O}_{3}$, respectively. It was certain that a series of complicated thermal chemical reactions had been produced at the interface during the heating process, which could be used to explain the unclear exothermic phenomenon as shown in Figure 3b. Additionally, the peak intensity of the individual spectral line directly represented the oxide content at the corrosion interface. It can be used to further determine the type of interfacial chemical reactions and study the oxidation wear process of single crystal diamond in depth.

\section{Conclusions}

In this work, a series of thermal analysis experiments between diamond specimens and steels under different conditions are performed to investigate single crystal diamond wear. The main conclusions obtained are summarized as follows:

(1) Diamond thermochemical wear includes the graphitization, diffusion and oxidation, and graphitization is the main wear mechanism. Temperature should be considered as the key factor influencing these wear mechanisms.

(2) The initial graphitization temperatures of diamond catalyzed by ferrous materials are obtained. The graphitized degree is observably intensified with the rising temperature, and it relies heavily on the diamond crystallographic plane while being insensitive to the work material.

(3) The diffusion wear rule is preliminarily achieved by the established prediction model, and this can be used as fundamental research for further investigation of diamond diffusion wear in combination with the reasonable experimental studies. Additionally, more attention should be paid to reducing the temperature and designing a diffusion barrier at the interface to suppress it.

(4) It is confirmed that the possibility of diamond reacting directly with iron to cause tool wear is relatively small by comparing with thermal analysis curves in different atmospheres. However, the presence of oxygen will lead to the occurrence of a large number of redox reactions at the interface, resulting in diamond oxidation wear.

This research can be used as the basis for expanding the application areas of ultra-precision machining with a single crystal diamond tool.

Acknowledgments: This work was supported by the National Natural Science Foundation of China (Grant No. 51605056) and the Research Funds for Basic Science and Advanced Technology of Chongqing (Grant No. cstc2016jcyjA0066).

Author Contributions: Lai Zou and Ming Zhou conceived and designed the experiments; Guijian Xiao performed the experiments; Lai Zou and Yun Huang analyzed the data; Yun Huang contributed the materials; Lai Zou wrote the paper. 
Conflicts of Interest: The authors declare no conflict of interest.

\section{References}

1. Cheung, C.F.; Lee, W.B. Characterisation of nanosurface generation in single-point diamond turning. Int. J. Mach. Tools Manuf. 2001, 41, 851-875. [CrossRef]

2. Fang, F.Z.; Venkatesh, V.C. Diamond turning of soft semiconductors to obtain nanometric mirror surfaces. Int. J. Adv. Manuf. Technol. 2002, 19, 637-641. [CrossRef]

3. Ramanujachar, K.; Subramanian, S.V. Micromechanisms of tool wear in machining free cutting steels. Wear 1996, 197, 45-55. [CrossRef]

4. Lane, B.M.; Dow, T.A.; Scattergood, R. Thermo-chemical wear model and worn tool shapes for single-crystal diamond tools cutting steel. Wear 2013, 300, 216-224. [CrossRef]

5. Venkatesh, V.; Swain, N.; Srinivas, G.; Kumar, P.; Barshilia, H.C. Review on the machining characteristics and research prospects of conventional microscale machining operations. Mater. Manuf. Process. 2016. [CrossRef]

6. Casstevens, J.M. Diamond turning of steel in carbon-saturated atmospheres. Precis. Eng. 1983, 5, 9-15. [CrossRef]

7. Hitchiner, M.P. Factors affecting chemical wear during machining. Wear 1984, 93, 63-80. [CrossRef]

8. Evans, C. Cryogenic diamond turning of stainless steel. CIRP Ann. Manuf. Technol. 1991, 40, 571-575. [CrossRef]

9. Song, Y.C.; Nezu, K.; Park, C.H.; Moriwaki, T. Tool wear control in single-crystal diamond cutting of steel by using the ultra-intermittent cutting method. Int. J. Mach. Tools Manuf. 2009, 49, 339-343. [CrossRef]

10. Shamoto, E.; Suzuki, N. Ultrasonic vibration diamond cutting and ultrasonic elliptical vibration cutting. Compr. Mater. Process. 2014, 11, 405-454.

11. Brinksmeier, E.; Gläbe, R.; Osmer, J. Ultra-precision diamond cutting of steel molds. CIRP Ann. Manuf. Technol. 2006, 55, 551-554. [CrossRef]

12. Inada, A.; Min, S.; Ohmori, H. Micro cutting of ferrous materials using diamond tool under ionized coolant with carbon particles. CIRP Ann. Manuf. Technol. 2011, 60, 97-100. [CrossRef]

13. Thornton, A.G.; Wilks, J. The wear of diamond tools turning mild steel. Wear 1980, 65, 67-74. [CrossRef]

14. Paul, E.; Evans, C.J. Chemical aspects of tool wear in single point diamond turning. Precis. Eng. 1996, 18, 4-19. [CrossRef]

15. Tanaka, H.; Shimada, S.; Ikawa, N.; Yoshinaga, M. Wear mechanism of diamond cutting tool in machining of steel. Key Eng. Mater. 2001, 196, 69-78. [CrossRef]

16. Shimada, S.; Tanaka, H.; Higuchi, M. Thermo-chemical wear mechanism of diamond tool in machining of ferrous metals. CIRP Ann. Manuf. Technol. 2004, 53, 57-60. [CrossRef]

17. Narulkar, R.; Bukkapatnam, S.; Raff, L.M.; Komanduri, R. Graphitization as a precursor to wear of diamond in machining pure iron: A molecular dynamics investigation. Comput. Mater. Sci. 2009, 45, 358-366. [CrossRef]

18. Zou, L.; Dong, G.J.; Zhou, M. Investigation on frictional wear of single crystal diamond against ferrous metals. Int. J. Refract. Met. Hard Mater. 2013, 41, 174-179. [CrossRef]

19. Jen, F.L.; Jia, W.L.; Pal, J.W. Thermal analysis for graphitization and ablation depths of diamond films. Diam. Relat. Mater. 2006, 15, 1-9.

20. Chen, Z.C.; Subhash, G.; Tulenko, J.S. Raman spectroscopic investigation of graphitization of diamond during spark plasma sintering $\mathrm{UO}_{2}$-diamond composite nuclear fuel. J. Nucl. Mater. 2016, 475, 1-5. [CrossRef]

21. Berman, R. The diamond-graphite equilibrium calculation: The influence of a recent determination of the Gibbs energy difference. Solid State Commun. 1996, 99, 35-37. [CrossRef]

22. Zou, L.; Zhou, M. Experimental investigation and numerical simulation on interfacial carbon diffusion of diamond tool and ferrous metals. J. Wuhan Univ. Technol.-Mater. Sci. Ed. 2016, 31, 307-314. [CrossRef]

23. Yamashita, T.; Hayes, P. Analysis of XPS spectra of $\mathrm{Fe}^{2+}$ and $\mathrm{Fe}^{3+}$ ions in oxide materials. Appl. Surf. Sci. 2008, 254, 2441-2449. [CrossRef] 